\title{
Transport of chaotic trajectories from regions distant from or near to structures of regular motion of the Fermi-Ulam model
}

\author{
Nilson B. de Faria, ${ }^{1}$ Daniel S. Tavares, ${ }^{1}$ Wenderson C. S. de Paula, ${ }^{1}$ Edson D. Leonel,${ }^{2}$ and Denis G. Ladeira ${ }^{1, *}$ \\ ${ }^{1}$ Departamento de Física e Matemática, UFSJ, Univ. Federal de São João del Rei, Rod. MG 443, Km 7, \\ Fazenda do Cadete, 36420-000 Ouro Branco, MG, Brazil \\ ${ }^{2}$ Departamento de Física, UNESP, Univ. Estadual Paulista, and Av. 24A, 1515 Bela Vista, 13506-900 Rio Claro, SP, Brazil
}

(Received 18 February 2016; revised manuscript received 28 August 2016; published 13 October 2016)

\begin{abstract}
The chaotic portion of phase space of the simplified Fermi-Ulam model is studied under the context of transport of trajectories in two scenarios: (i) the trajectories are originated from a region distant from the islands of regular motion and are transported to a region located at a high portion of phase space and (ii) the trajectories are originated from chaotic regions around the islands of regular motion and are transported to other regions around islands of regular motion. The transport is investigated in terms of the observables histogram of transport and survival probability. We show that the histogram curves are scaling invariant and we organize the survival probability curves in four kinds of behavior, namely (a) transition from exponential decay to power law decay, (b) transition from exponential decay to stretched exponential decay, (c) transition from an initial fast exponential decay to a slower exponential decay, and (d) a single exponential decay. We show that, depending on choice of the regions of origin and destination, the transport process is weakly affected by the stickiness of trajectories around islands of regular motion.
\end{abstract}

DOI: 10.1103/PhysRevE.94.042208

\section{INTRODUCTION}

Escape, transmission, reflection, and recurrence time statistics are some of the approaches employed to study properties of trajectories in phase space of dynamical systems. Escape processes are usually studied in the context where a system presents a natural leak or hole through which a particle or a wave leaves the system [1-6]. A leak or hole can be introduced in closed systems in order to study the properties of trajectories in phase space [7-9] or to simulate loss in confinement of plasma and measurement devices, e.g., antennas and sensors [10-13]. Transport is the process by which trajectories initially located at a region of origin evolves to a destination region. The location of regions of origin and destination is arbitrary and depends on the subject of interest under study. The properties of transport and escape processes are dependent on the nature of the system under investigation.

Nonlinear Hamiltonian systems usually present phase space composed of a coexistence of regular and chaotic trajectories. Depending on the system, the regions of regular motion are formed by complex structures of Kolmogorov-ArnoldMoser (KAM) islands with positive measure [7,14,15]. Other systems, e.g., the mushroom and stadium billiards [16-23], also present the so-called marginally unstable periodic orbits. They form families of orbits that remain trapped forever. The most simple set of such dynamics corresponds to the situation where the particle collides perpendicularly between parallel walls. It is also worth mentioning that under certain conditions the mushroom billiard presents sharply divided phase space $[22,24]$. For systems that present phase space with strong chaotic properties, observables such as Poincaré recurrence times and survival probability decay exponentially $[6,25,26]$. Chaotic trajectories evolving in phase space are deeply affected by the presence of regions of regular motion. Eventually, these

*Corresponding author: dgl@ufsj.edu.br chaotic trajectories come close enough to a KAM island and stick to the border of this regular region for long periods of time $[27,28]$. As a consequence, in a system with phase space composed by mixed structure, i.e., regions of chaotic motion coexisting with regular trajectories, the Poincare recurrence times and survival probability present an initial regime of exponential decay followed by a slower decay described by power law [8,9] or stretched exponential [29]. These slower-than-exponential decays are attributed to the temporary trapping, or stick, of chaotic trajectories near KAM islands or other periodic regions. Due to stickiness, properties such as transport [30] and decay of correlations [27,28] are affected even by small-size islands. When a region [31-34] encloses a pair of broken separatrices, the lobes formed by manifolds and homoclinic points form structures in phase space that work as turnstiles, i.e., places where trajectories arrive and leave the resonance zones. Zaslavsky [30] used renormalization to chains of self-similar islands and derived relations between the exponent of the power-law decay and scaling exponents. Chirikov and Shepelyansky proposed renormalization arguments at the breakdown of the golden mean torus to describe the stickiness of trajectories [35] in the standard map. Moreover, the nonuniform distribution of trajectories in mixed phase spaces and the stick domains had been studied in the context of anomalous transport [30,36-38]. Although the development of the study of transport since the mid-1980s is notable, several questions are still unanswered. As pointed by Meiss [33], some of these questions involve the possibility of using the available flux formulas to calculate the flow of trajectories through two-dimensional manifolds in threedimensional maps [39-41] and the topology of the structures that replace invariant torus in multidimensional symplectic maps. Moreover, the cantori remnant of the destruction of tori work as partial barriers and the flux is locally minimal [39]. So another unanswered question is related to the monotonicity of asymptotic flux in analytic maps. 
In this work we study the transport of chaotic trajectories in the Fermi-Ulam model. In particular, we are interested in understanding and describing the transport dynamics in two different situations: (a) the scenario where the region of origin is located distant from regions of regular motion and (b) situation where the regions of origin are around islands of regular motion. This investigation contributes to the understanding of transport in conservative systems and our findings and tools can be extended to other classes of dynamical systems such as plasma confinement systems as well as billiard problems. This paper is organized as follows. In Sec. II, we present the model, the approach, and the observables used in the study of transport process. In Sec. III we discuss the results and in Sec. IV we present the conclusions of the present study.

\section{TRANSPORT PHENOMENA}

In this work we study the transport process of chaotic trajectories in the simplified Fermi-Ulam model. This model consists of a pair of massive walls that confine a particle. One of the walls oscillates in time, $t^{\prime}$, according to the expression $x\left(t^{\prime}\right)=\epsilon \cos \left(\omega t^{\prime}+\phi_{0}\right)$ and transfers energy and momentum to the particle at each collision. The symbol $\epsilon$ represents the amplitude of motion of the moving wall, $\omega$ is the frequency, and $\phi_{0}$ denotes an initial phase. The other wall is fixed at position $x=l$ and works as a returning mechanism that brings the particle back to the moving wall. The collisions are elastic and the particle moves freely between the walls. Defining the set of dimensionless variables $X=x / l, t=\omega t^{\prime}$, and $V=v / \omega l$, $\phi=t+\phi_{0}$, we have that the velocity of particle and phase of moving wall after each collision with the moving wall is given by

$$
T:\left\{\begin{array}{l}
\phi_{n+1}=\left(\phi_{n}+2 / V_{n}\right) \quad \bmod 2 \pi \\
V_{n+1}=\left|V_{n}-2 \varepsilon \sin \phi_{n+1}\right|
\end{array}\right.
$$

where $\varepsilon=\epsilon / l$ is the dimensionless parameter and the absolute value bars prevent the particle to live out the confinement. It is important to mention that $\varepsilon$ controls a transition from integrable, when $\varepsilon=0$, to nonintegrable, when $\varepsilon \neq 0$. Figure 1 illustrates the phase space of Fermi-Ulam model for three different values of the control parameter, namely $\varepsilon=10^{-4}, 10^{-3}, 10^{-2}$.

Transport is a process by which trajectories initially located at a predefined region, or window, of phase space evolve to other predefined window of phase space. The study of transport we focus on here consists iof the investigation of the following observables: (i) normalized histograms of transport and (ii) survival probability. Let us describe each of these observables separately.

Once defined a window of origin, $W_{o}$, and a window of destination, $W_{d}$, the window $W_{o}$ is filled with a set of $M$ initial conditions (ICs). Then a numerical code evaluates the trajectory of each IC inside $W_{o}$ using the map (1) until one of the following conditions is satisfied: (i) the trajectory of this IC reaches the destination window $W_{d}$ or (ii) the trajectory evolves a maximum number of iterations, $n_{\max }$, without reaching $W_{d}$. Then the evaluation of this trajectory is interrupted and the iteration of other IC starts. This procedure repeats until the ensemble of $M$ ICs is totally exhausted.

The histogram $H(n)$ is the number of trajectories reaching the destination window at iteration $n$. Numerically, $H(n)$ is evaluated according to the following procedure. An array $H$ with $n_{\max }+1$ elements is created. Before starting the iterative process of the ICs, $H$ is set to zero for all components, from $i=0$ until $i=n_{\max }$. Then the iterative procedure of the ICs starts. If the trajectory of an IC reaches $W_{d}$ at iteration $i$, then the component $i$ of $H$ is increased by unity and the trajectory of other IC is started. At the end of $M \mathrm{ICs}$, the array $H(n)$ has the distribution of the number of transported trajectories at each value of $n$. Because $H(n)$ depends on the size $M$ of the ensemble of ICs inside $W_{o}$, it defines the quantity

$$
h(n)=\frac{H(n)}{M},
$$

which is the normalized histogram of transport.

To define the survival probability, let us describe $N(n)$ as the amount of trajectories that do not reach $W_{d}$ until the iteration $n$. At the beginning of simulation, we have $N(0)=M$, since all trajectories (ICs) are inside $W_{o}$. Eventually, as described above, during the iterative process, a trajectory reaches $W_{d}$ and the numerical evaluation of it is terminated. If it happens at iteration $i$, then the quantity $N(n)$ decreases by unity for $i \leqslant n \leqslant n_{\max }$ and the trajectory of other IC starts. So, as the numerical simulation evolves, the number of remaining trajectories gradually decreases. Consequently, at the end of simulation we have that the quantity $N(n)$ is a decreasing function. The survival probability, $P(n)$, is the normalized quantity defined by

$$
P(n)=\frac{N(n)}{M} .
$$

The large the $M$, the smother the curve $P(n)$.

\section{RESULTS AND DISCUSSION}

In this section we present the results of transport of chaotic trajectories considering two scenarios. In Sec. III A we present the results obtained regarding the window of origin, $W_{o}$, as a region in the chaotic sea located distant from the structures of regular motion in the phase space of the Fermi-Ulam model. The destination window, $W_{d}$, is defined as a region of chaotic sea located in phase space in terms of the average position of the lowest-energy-spanning curve of the Fermi-Ulam model. In Sec. III B we present the results of the transport process where $W_{o}$ and $W_{d}$ are narrow portions of chaotic sea around islands of regular motion.

\section{A. Windows of origin distant from regions of regular motion}

Let us describe how the windows of origin and destination, $W_{o}$ and $W_{d}$, are defined. The phase space of the Fermi-Ulam model presents a region of global chaos that surrounds islands of regular motion. These islands are observed at high and intermediate portions of the chaotic region. For values of velocity below the intermediate region of the chaotic sea there are no regions of regular motion since the periodic points are unstable [42]. In order to define a window of origin distant from 
the regular motion structures, we defined $W_{o}$ as the portion of chaotic sea located at $V=10^{-3}$ and $\phi \in[0, \pi)$.

To define the destination window we need to first discuss how the location of the invariant spanning curve of lowest energy was obtained. We started defining an array of size $10^{4}$ where each component corresponds to a narrow stripe in phase space as a result of the division of the interval of phase $[0,2 \pi$ ) in $10^{4}$ parts of same measure, and we attributed the initial value zero for all components of this array. Then we used an initial condition located in the chaotic sea and iterated the map (1). With the value of the phase after each iteration we determined the stripe where the point of the trajectory was located. If the value of velocity was greater than the value stored at the corresponding stripe of phase, then we replaced the previous value by the current value of velocity. Otherwise, the vector remains unchanged. By performing this procedure after each iteration for a long-enough simulation, we obtain a good approximation for the location of the lowest-energyspanning curve. The greater the number of iterations, the better the approximation. The size of the chaotic sea and, therefore, the location of the spanning curve of lowest energy depends on $\varepsilon$. So we obtained the approximated spanning curves of lowest energy for 19 values of parameter $\varepsilon$ in the interval $\left[10^{-4}, 10^{-2}\right]$. These approximations to the spanning curves were obtained after we iterated $10^{12}$ times the map (1) for each $\varepsilon$. Because the value of velocity in an invariant spanning curve is not a constant, we evaluated the average velocity $\langle V\rangle_{\text {inv }}$. Then we defined $W_{d}$ at the high portion of the chaotic sea as the region of phase space above the line $V_{c}=c\langle V\rangle_{\text {inv }}$ and below the invariant curve of lowest energy. We use the fixed value $c=0.75$, although other values of $c$ can also be used. In Ref. [43] the authors discussed the transport process in a time-dependent potential well. They showed that the value of $V_{c}$ changes the time until the particle reaches such a value but the universal properties are unchanged for different values of $c$.

Once $W_{o}$ and $W_{d}$ are established, the next step consists of determining the ICs in $W_{o}$. So we defined an ensemble of $M=10^{8}$ ICs with velocity $V_{0}=10^{-3}$ and initial phases $\phi_{0}$ randomly chosen in the interval $\phi \in[0, \pi)$.

In this section we examine the properties of transport of chaotic trajectories studying the observables histogram of transport and survival probability presented in Sec. II. In both cases we used $n_{\max }=10^{7}$. So let us start the discussion presenting the results related to the histograms.

Figure 2 displays the histogram $h(n)$ for five different values of parameter $\varepsilon$, as shown in the legend. For each curve we observe that $h(n)$ is an increasing function for small-enough $n$. This is expected because after the initial iterations most of the trajectories are still at the low portion of the phase space and few trajectories reach $W_{d}$. But while iterations proceed, more and more trajectories acquire net energy gain and reach the destination window $\left(V>V_{c}\right)$. Moreover, we observe in Fig. 2 that each $h(n)$ curve reaches a maximum value and after that becomes a decreasing function. This decay is expected because the bigger the number of iterations, the smaller the fraction of remaining trajectories in simulations.

Let us designate $h_{\max }$ as the maximum value of each $h(n)$ curve and $n_{p}$ as the value of $n$ for which $h\left(n_{p}\right)=h_{\max }$. So $n_{p}$ represents the crossover from the growth regime to the decay regime of the normalized histogram $h(n)$. As we observe in Fig. 2, the quantities $h_{\max }$ and $n_{p}$ depend on $\varepsilon$. Specifically,
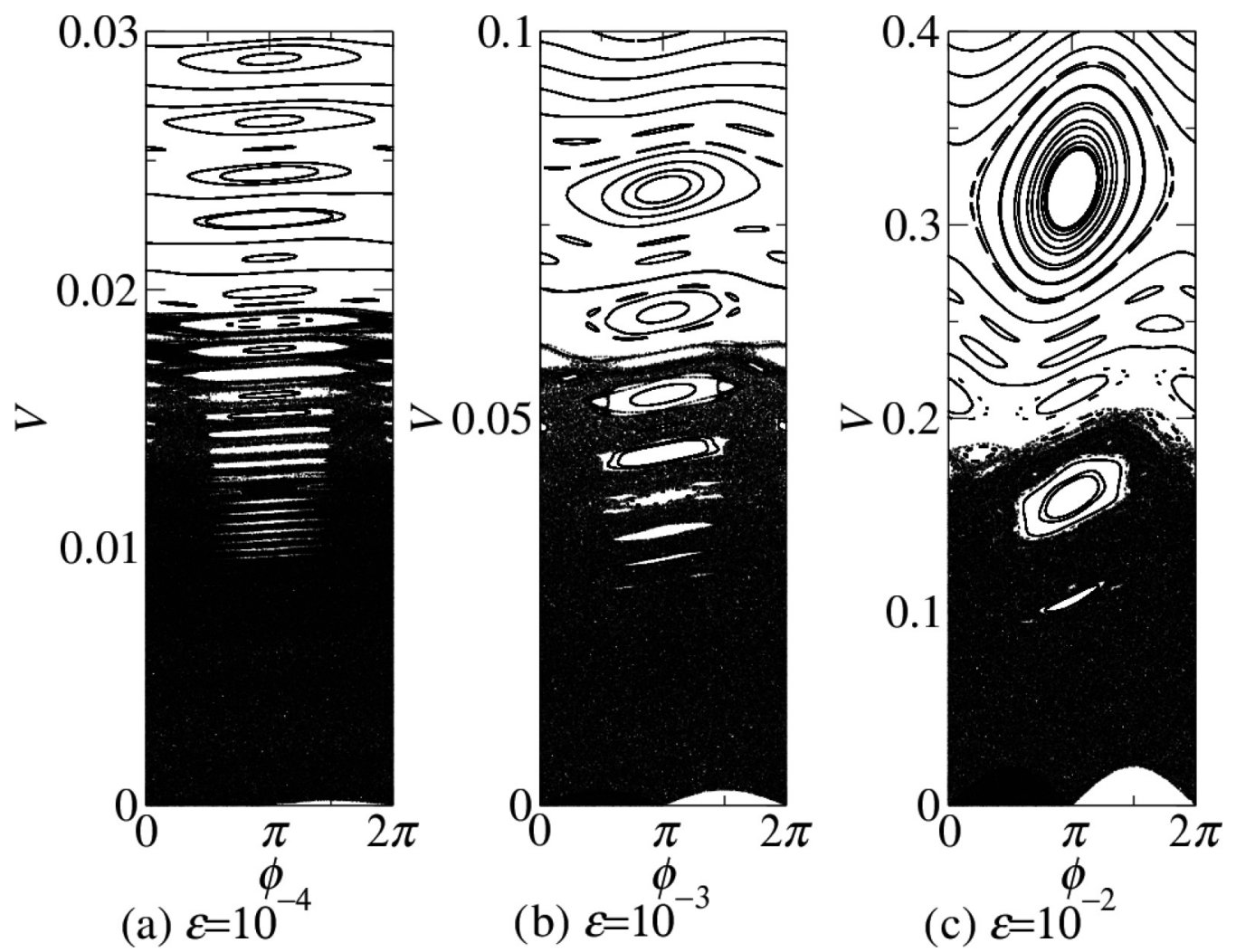

FIG. 1. Phase space of Fermi-Ulam model for (a) $\varepsilon=10^{-4}$, (b) $\varepsilon=10^{-3}$, and (c) $\varepsilon=10^{-2}$. 
when we increase the parameter $\varepsilon$ the maximum $h_{\text {max }}$ increases while the value of $n_{p}$ decreases.

Near $n_{p}$ the curves of $h(n)$ are described by a quadratic function with a good approximation. So, with the parameters of quadratic fits we obtained the coordinates $\left(n_{p}, h_{\max }\right)$ of the transition from the growth to decay regimes for each $h(n)$ curve. Figure 3(a) illustrates the log-log plot of the crossover $n_{p}$ as function of $\varepsilon$. The best fit to the numerical data furnishes $n_{p} \propto \varepsilon^{\gamma}$ with negative exponent $\gamma=-1.00 \pm 0.03$. We show in Fig. 3(b) the plot of $h_{\max }$ for several values of parameter $\varepsilon$ in $\log$ - $\log$ scales. The best fit to the numerical data provides that $h_{\max } \propto \varepsilon^{\zeta}$ with $\zeta=0.99 \pm 0.05$. The signals of exponents $\gamma$ and $\zeta$ are in agreement with our discussion in the previous paragraph.

With this information we can formally present the scaling description of $h(n)$. The numerical data suggest that the normalized histogram is described by the following homogeneous function

$$
h(n, \varepsilon)=\operatorname{lh}\left(l^{a} n, l^{b} \varepsilon\right),
$$

where $l$ is a scaling factor and $a$ and $b$ are scaling exponents. Choosing $l=\varepsilon^{-1 / b}$, the above expression becomes

$$
h(n, \varepsilon)=\varepsilon^{-1 / b} f\left(\varepsilon^{-a / b} n\right),
$$

where $f\left(\varepsilon^{-a / b} n\right)=h\left(\varepsilon^{-a / b} n, 1\right)$. For $n \approx n_{p}$ we have $h \approx$ $h_{\max }$ and, therefore, $h_{\max } \propto \varepsilon^{-1 / b}$. With this result and from the discussion about the numerical results, we have that the critical exponent $\zeta$ and the scaling exponent $b$ are related by $\zeta=-1 / b$ and, therefore, $b=-1.01 \pm 0.05$. Moreover, for $n \approx n_{p}$ we obtain from the above expression that $n_{p} \propto \varepsilon^{a / b}$. Therefore the critical exponent $\gamma$ and the scaling exponents $a$ and $b$ are related by $\gamma=a / b$. With this relation and the value of $b$ we obtain the scaling exponent $a=1.01 \pm 0.08$. Performing the variable transformations $h \rightarrow h / \varepsilon^{-1 / b}$ and $n \rightarrow n \varepsilon^{-a / b}$ we obtain the collapse of $h$ curves of Fig. 2 into a single and universal curve, as illustrated in Fig. 4.

Let us now address the survival probability $P(n)$ as function of $n$. Figure 5 illustrates the survival probability curves for five values of $\varepsilon$. Figure 5(a) is a $\log$-linear plot of $P(n)$ versus $n$ for small values of $n$, where we observe an exponential decay. Figure 5(b) is a log-log plot of the survival probability. The

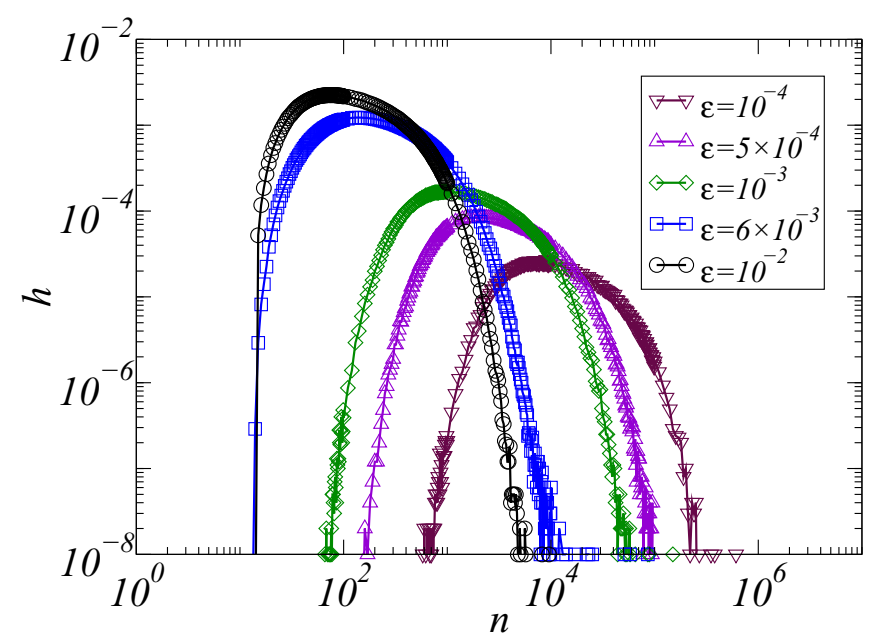

FIG. 2. Histograms of transport for different values of parameter $\varepsilon$.
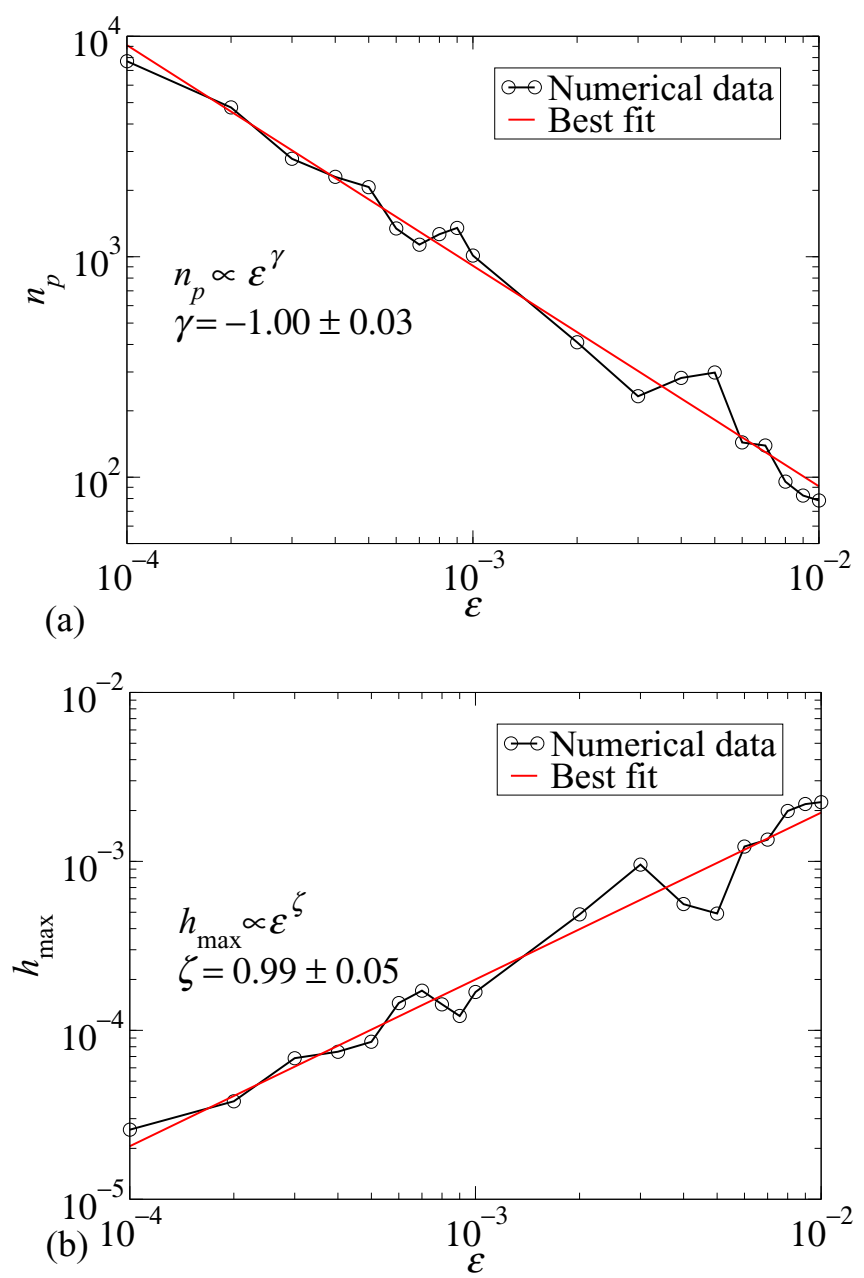

FIG. 3. (a) The crossover $n_{p}$ as a function of $\varepsilon$ and (b) the maximum value of each histogram versus $\varepsilon$.

power-law decay observed for large values of $n$ is consistent with the existence of sticky domains in the phase space. These sticky domains are regions located around islands of regular

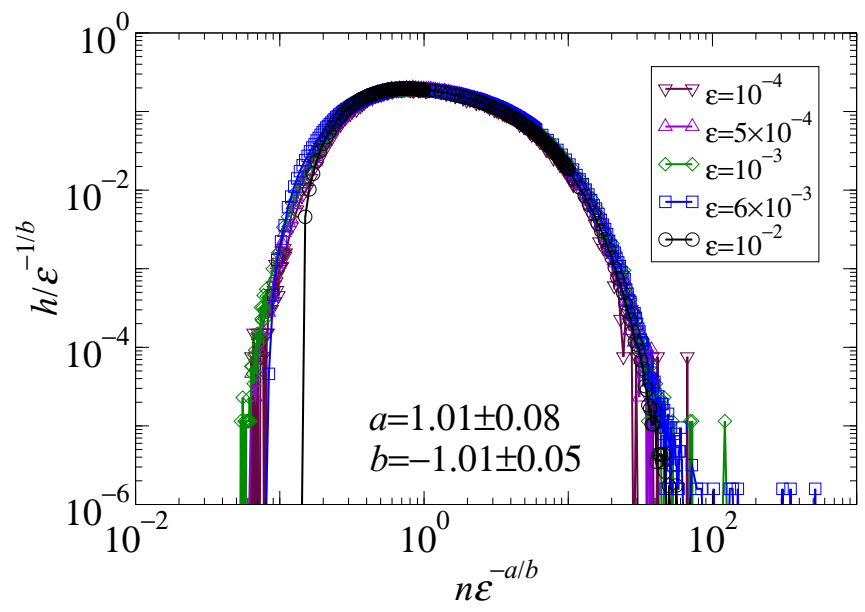

FIG. 4. The appropriate scale transformations overlap the different curves of the histograms into a single and hence universal curve. 

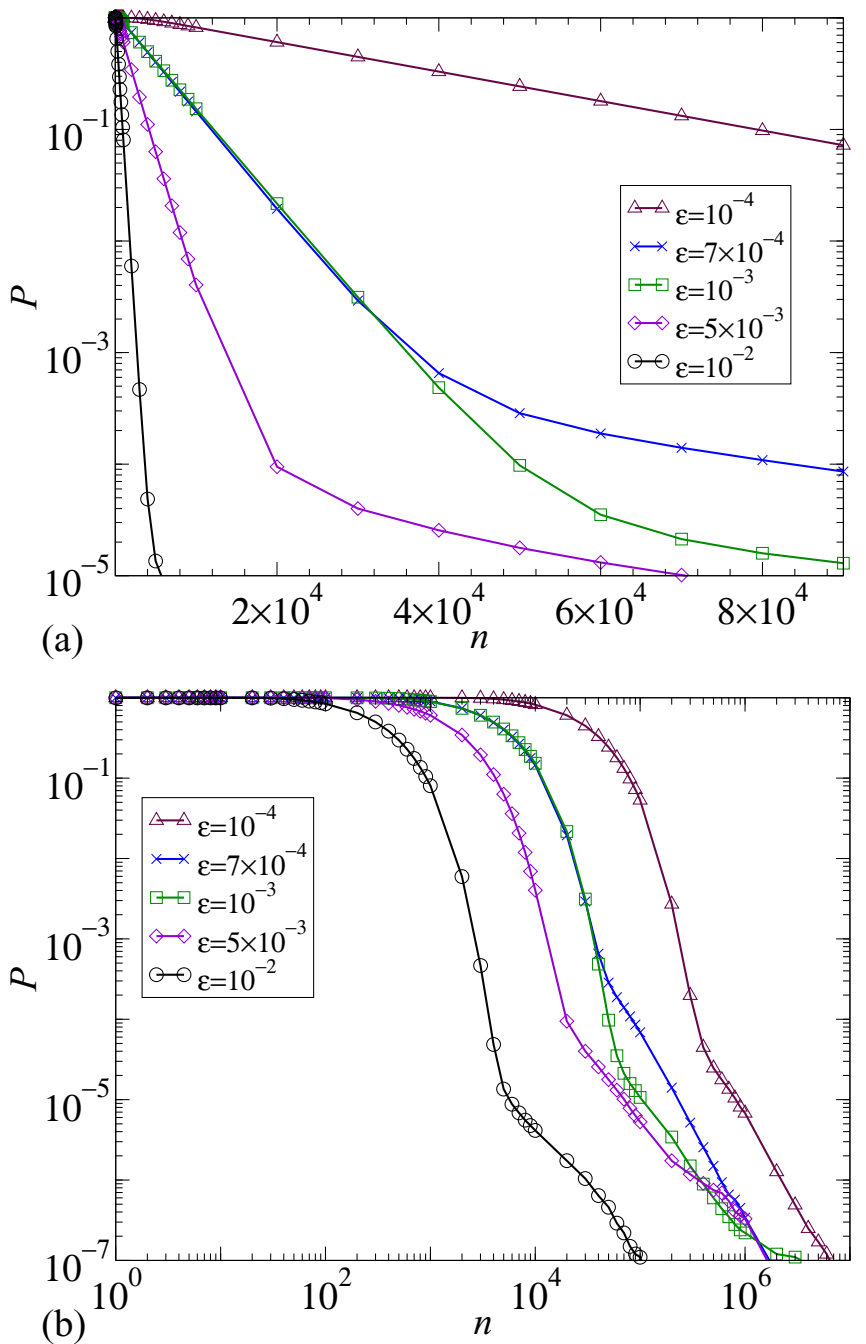

FIG. 5. (a) Log-linear plot of survival probability as function of $n$ illustrates the exponential decay observed for small values of $n$. (b) Log-log plot of survival probability illustrates power-law decays for big values of $n$.

motion and near invariant curves, where chaotic trajectories are eventually trapped for a long time. As a consequence, the survival probability presents a decay slower than exponential, typically a power law or a stretched exponential $[8,9,29]$.

We define $n_{c}$ as the value of $n$ which characterizes the changeover from the exponential decay to the power-law decay. We observe that the value of $n_{c}$ decreases when $\varepsilon$ increases. Performing fits to the numerical data in the regimes $n \ll n_{c}$ and $n \gg n_{c}$ and using direct algebra, we obtain the value of $n_{c}$ for each curve. Figure 6(a) shows a log-log plot of $n_{c}$ versus $\varepsilon$. The best fit to the data furnishes $n_{c} \propto \varepsilon^{\beta}$ with $\beta=-1.00 \pm 0.06$. Performing the scale transformation $n \longrightarrow n / \varepsilon^{\beta}$ in the curves of Fig. 5, we obtain the universal behavior of the initial decay of the survival probability, as depicted in Fig. 6(b).

It is known that the position of the lowest-energy invariant spanning curve in phase space of the Fermi-Ulam model obeys the relation $\langle V\rangle_{\text {inv }} \propto \varepsilon^{1 / 2}[44,45]$. Because $V_{c}$ is proportional to $\langle V\rangle_{\text {inv }}$, then $V_{c} \propto \varepsilon^{1 / 2}$. As discussed before, the crossover $n_{p}$ that characterizes the change from the growth to the decay
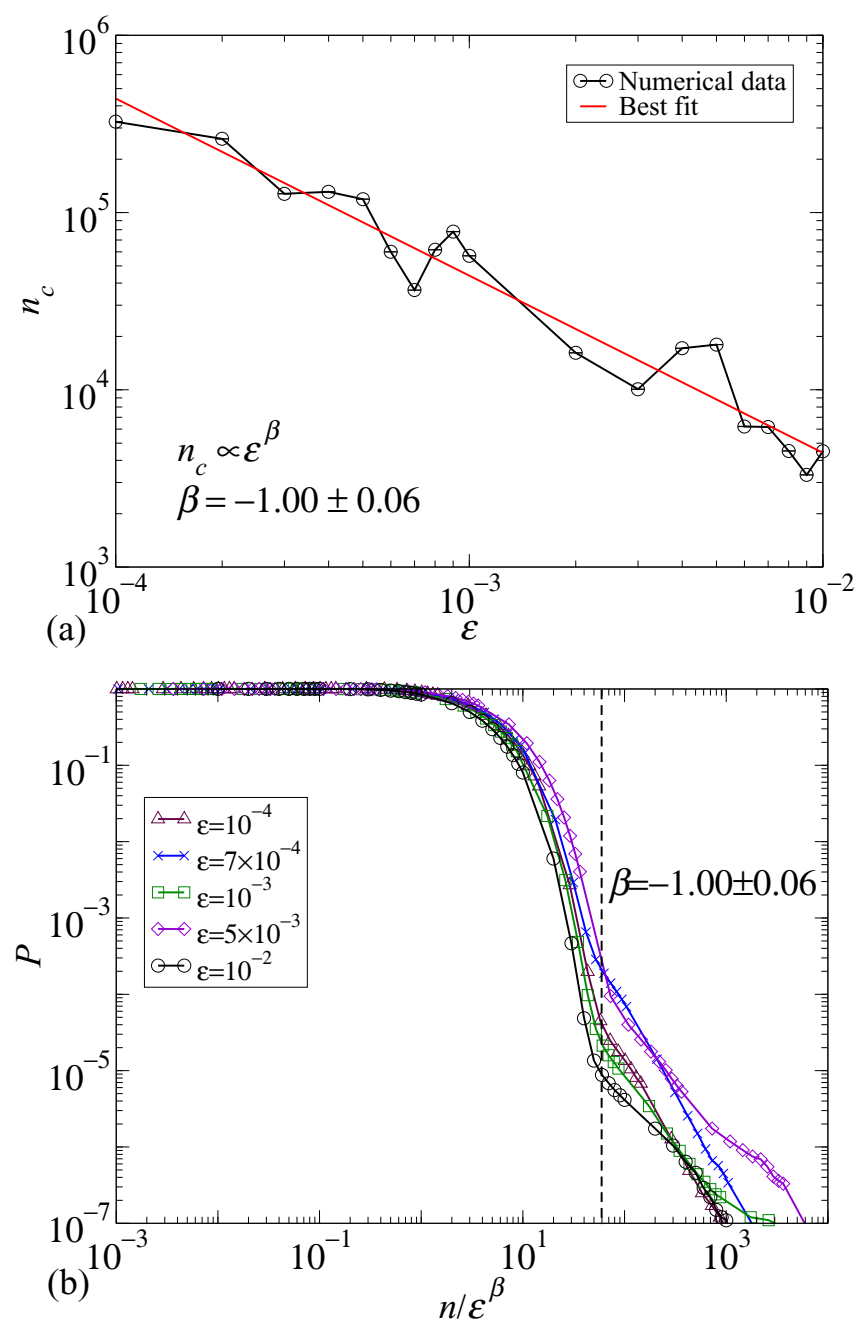

FIG. 6. (a) Log-log plot of crossover value $n_{c}$ of survival probability as function of $\varepsilon$. (b) Collapse of survival probability curves in a single curve after a rescaling.

regime of $h$ curves and the crossover $n_{c}$ that marks the transition from exponential to power-law decay of the survival probability curves are, respectively, related to $\varepsilon$ by $n_{p} \propto \varepsilon^{\gamma}$ and $n_{c} \propto \varepsilon^{\beta}$, where $\gamma \approx \beta \approx-1$. Comparing the values of $n_{p}$ and $n_{c}$, Figs. 3(a) and 6(a), we observe that $n_{c}$ is near two order of magnitude greater than $n_{p}$. It means that maximum transport rate occurs before the transition from exponential to power law decay. Moreover, we observed that the maximum value of the histogram curves depends on $\varepsilon$ according to $h_{\max } \propto \varepsilon^{\zeta}$ with $\zeta \approx 1$. Therefore we have that $h_{\max } \propto V_{c}^{2}$ and $n_{p} \propto V_{c}^{-2}$. Because of these two results, we conclude that $h_{\max } \propto \varepsilon^{1}$ and $n_{p} \propto \varepsilon^{-1}$, as confirmed numerically.

\section{B. Windows of origin and destination around regions of regular motion}

In this section we describe transport processes of chaotic trajectories from windows of origin $W_{o}$ located around islands of regular motion associated to period-1 fixed points to destination windows $W_{d}$ located around other islands of regular motion as we describe now. 
TABLE I. Parameters that define the windows of origin and destination for (a) $\varepsilon=10^{-3}$ and (b) $\varepsilon=10^{-4}$.

\begin{tabular}{lccc}
\hline \hline$m$ & $\mu$ & $e$ & $\alpha$ \\
\hline & & $(\mathrm{a})$ & \\
5 & 2.2 & $1-1.2 \times 10^{-6}$ & $2.1 \pi \times 10^{-4}$ \\
6 & 1.9 & $1-9 \times 10^{-7}$ & $2.5 \pi \times 10^{-4}$ \\
9 & 1.3 & $1-2.2 \times 10^{-7}$ & $2.2 \pi \times 10^{-4}$ \\
10 & 1.2 & $1-6 \times 10^{-8}$ & $2.7 \pi \times 10^{-4}$ \\
& & $(\mathrm{~b})$ & \\
16 & 2.4 & $1-1.4 \times 10^{-6}$ & $1.72 \pi \times 10^{-5}$ \\
17 & 2.2 & $1-1.4 \times 10^{-8}$ & $1.8 \pi \times 10^{-5}$ \\
31 & 1.3 & $1-8.5 \times 10^{-10}$ & $2.7 \pi \times 10^{-5}$ \\
32 & 1.5 & $1-4.4 \times 10^{-10}$ & $2.7 \pi \times 10^{-5}$ \\
\hline \hline
\end{tabular}

The fixed points of the simplified Fermi-Ulam model are located at the coordinates $(\phi, V)=\left(\phi^{*}, V^{*}\right)$ with $\phi^{*}=$ $(0, \pi)$ and $V^{*}=1 /(m \pi)$ for $m=1,2,3, \ldots$ The fixed points associated to $\phi^{*}=0$ are unstable. Therefore we focus in the islands of regular motion observed around the fixed points associated to $\phi^{*}=\pi$. These islands of regular motion are surrounded by a chaotic sea. Because our purpose is to study the transport from $W_{o}$ to $W_{d}$ in the chaotic sea around islands of regular motion, we define elliptical windows that enclosure a thin layer of chaotic sea that surrounds the regions of regular motion. So we use polar variables $\rho$ and $\theta$ to represent the coordinates $(x, y)$ of the border of the ellipses as $x=\rho \cos \theta$ and $y=\rho \sin \theta$, where $\rho=\mu \sigma / \sqrt{(\sigma \cos \theta)^{2}+(\mu \sin \theta)^{2}}$. The parameters $\mu$ and $\sigma$ represent the semiaxes of the ellipses. In terms of $\mu$ and the eccentricity $e$, the parameter $\sigma$ is given by $\sigma=\mu \sqrt{1-e^{2}}$. It is observed that the regions of regular motion present inclinations around a horizontal axis. Therefore we use the rotation matrix to gyrate the ellipses by an angle $\alpha$ measured with respect to the horizontal. This procedure furnishes the coordinates of a rotated ellipse, given by $x_{r}=$ $x \cos \alpha-y \sin \alpha$ and $y_{r}=x \sin \alpha+y \cos \alpha$. Displacing the center of the rotated ellipse from the origin to the position of the fixed points, the coordinates of the ellipses in the plane $\phi$ vs. $V$ are $\phi=x_{r}+\pi$ and $V=y_{r}+1 /(m \pi)$.

The smaller the $m$, the greater the size of the KAM island. Moreover, each island presents a different slope with respect to a horizontal line. Therefore we used values of parameters $\mu, e$, and $\alpha$ that furnish the ellipses a best adjust to the narrow layers of the chaotic sea that surround the islands. The Table I(a) summarizes the values of the parameters of these elliptical windows for $\varepsilon=10^{-3}$ for each value of $m$ used in this work. Similarly, Table I(b) presents the parameters of the windows for $\varepsilon=10^{-4}$ for the corresponding values of $m$.

The values of $m$ presented in Table I were chosen based in our interest in the study of transport from $W_{o}$ to $W_{d}$ combining situations where $W_{o}$ and $W_{d}$ could be close to or distant from each other.

We define $W_{m}$ as the elliptical window that incorporates a thin layer of the chaotic sea around the region of regular motion with center at the elliptical fixed point associated to the integer $m$. This is the procedure we used to define the windows of origin and destination which we use to study the
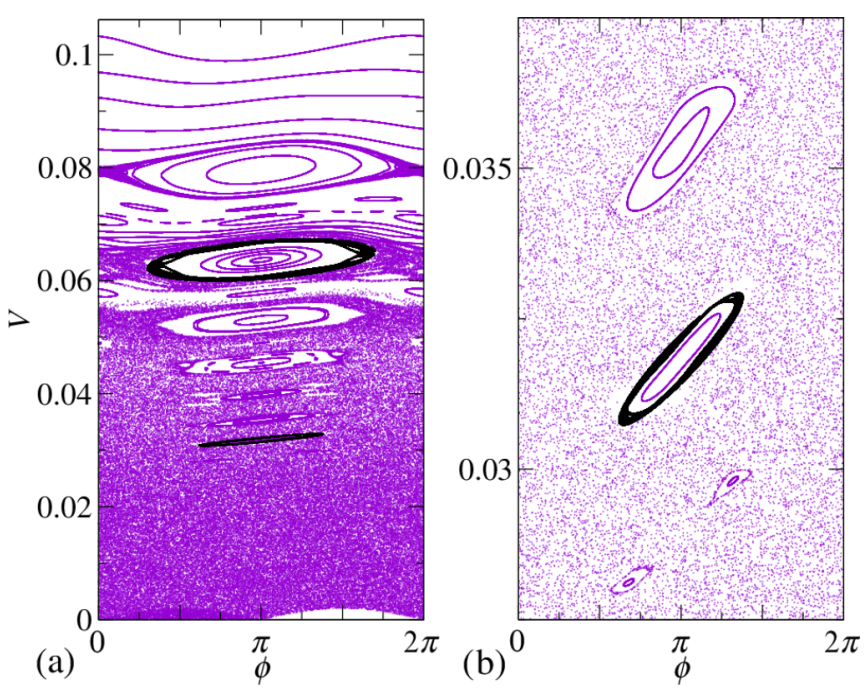

FIG. 7. (a) Phase space of model in gray (violet); ICs chosen in the chaotic sea inside $W_{5}$ and $W_{10}$ in black. (b) Amplification of region around window $W_{10}$.

transport process between two regions located near islands of regular motion.

To define the ICs inside $W_{o}$, for a predefined $o=m$, we used the following procedure. An IC located in the chaotic sea is iterated by the map (1). Eventually, the trajectory enters in the window $W_{o}$. When that happens, we store the coordinates of the trajectory first by truncating the values of $\phi$ and $V$ at the eighth significant digit. This truncation is enough to avoid considering different points of a same trajectory in the posterior transport study. After we stored a predefined number $M$ of points inside $W_{o}$ the procedure finishes. This set of points is used as the ICs inside the window of origin.

Figure 7 illustrates the phase space of the Fermi-Ulam model for $\varepsilon=10^{-3}$. The gray points (violet) correspond to the trajectories, including chaotic and regular ones, obtained by iteration of map (1). The black points correspond to ICs, inside the elliptical windows $W_{5}$ and $W_{10}$, obtained by the procedure described in the previous paragraph. Figure 7 (b) is an amplification of Fig. 7(a) where the ICs in the portion of the chaotic sea inside the window $W_{10}$ is illustrated in black.

Defining the ensemble of $M$ ICs inside a $W_{o}$, we specify a $W_{d}$, with $d \neq o$, and the transport process from $W_{o}$ to $W_{d}$ is evaluated as described in Sec. II. The number $M$ of ICs and the maximum iteration number $n_{\max }$ changed according to the necessity of each simulation. So in simulations we used $M=10^{5}, M=10^{6}$, or $M=10^{7}$ and $n_{\max }=10^{7}$ or $n_{\max }=$ $10^{8}$. In this section we focus in the study of properties of survival probability. The results we now discuss are organized according to the behavior of $P(n)$ in four different ways.

Figure 8 illustrates the survival probability related to the transport of trajectories from different windows of origin to different windows of destination for $\varepsilon=10^{-4}$ and $\varepsilon=10^{-3}$. Figure 8(a) illustrates, in log-log scales, the power-law decay of $P(n)$ in the regime of big values of $n$ for the transport from $W_{16}$ to $W_{17}$ and from $W_{16}$ to $W_{32}, \varepsilon=10^{-4}$, and from $W_{10}$ to $W_{9}$ for $\varepsilon=10^{-3}$. The long-term regime of transport from $W_{6}$ to $W_{5}$ and from $W_{10}$ to $W_{5}, \varepsilon=10^{-3}$, is characterized by 

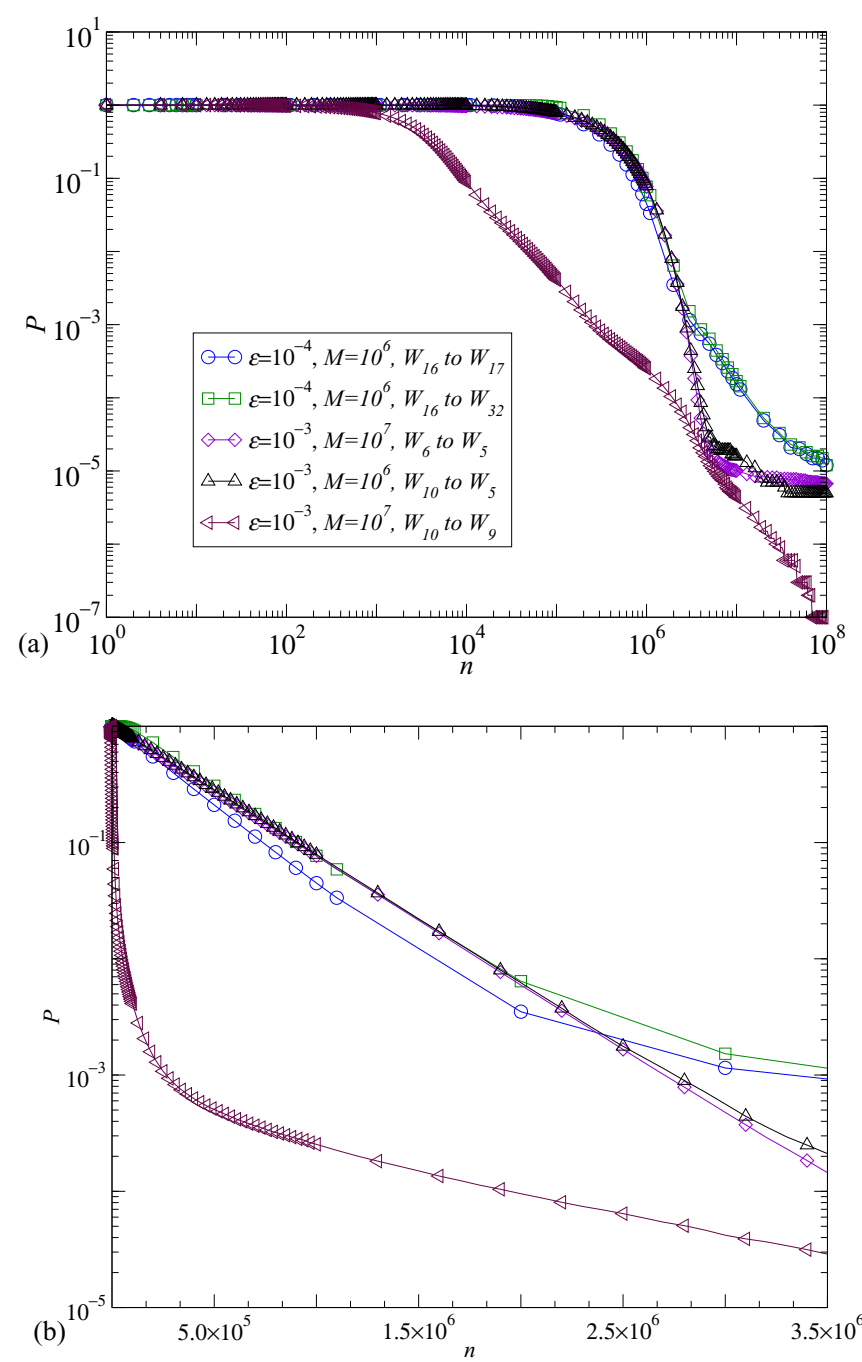

FIG. 8. (a) Log-log plot of survival probability of transport between different windows for different values of $\varepsilon$. (b) Log-linear plots of the initial regime of decay for the curves displayed in (a).

a very slow decay, apparently a power law. However, at this stage, the reduced number of decades and the relative small number of remaining trajectories does not allow us to define precisely the decay form for large values of $n$. This very slow decay is attributed to a few trajectories that remain stuck in regular motion structures for very long times. In Fig. 8(b) we present the plot of $P(n)$ in log-linear scales, where we see the exponential decay of $P(n)$ for small values of $n$. In the particular case of $P(n)$ for $\varepsilon=10^{-3}$ from $W_{10}$ to $W_{9}$ the exponential decay is observed for values of $n$ up to $6 \times$ $10^{3}$. The initial exponential decay of $P(n)$ is attributed to the contribution of chaotic sea, whereas the power-law decay is related to the trajectories temporarily trapped near regions of regular motion $[8,9]$.

A close examination of the $P(n)$ curves in Fig. 8 reveals that the transport from $W_{16}$ to $W_{17}$ and from $W_{16}$ to $W_{32}, \varepsilon=10^{-4}$, although similar, presents values of $P(n)$ that are greater for the second case. This is expected because the window of origin is the same for both cases (as well as the ICs) but the destination window is far apart for the second case. A similar situation

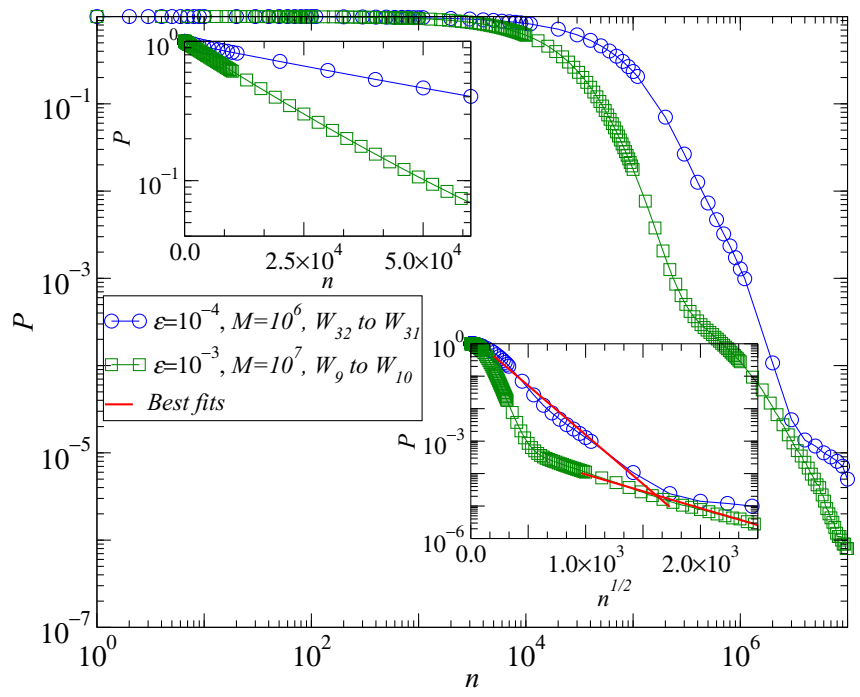

FIG. 9. Plot of survival probability as function of $n$.

occurs for the transport from $W_{6}$ to $W_{5}$ and from $W_{10}$ to $W_{5}$, regarding $\varepsilon=10^{-3}$, for the most values of $n$ in the $P(n)$ curves. Now the destination window is the same in both cases and the origin window differs. Only near the end of simulation is $P(n)$ greater for the transport from $W_{6}$ to $W_{5}$ than from $W_{10}$ to $W_{5}$. This is a consequence of two factors. The first is the small number of remaining trajectories near the end of simulation in both transports. In the transport from $W_{6}$ to $W_{5}$, the values of $P(n)$ correspond to 70 trajectories, or $7 \times 10^{-4} \%$ of the ICs, while in the transport from $W_{10}$ to $W_{5}$ the values of $P(n)$ near the end of simulation correspond to six trajectories, or $6 \times 10^{-4} \%$, of the ICs. The second factor is the fact that the ensembles of ICs differ in each transport. These two factors combined lead us to conclude that the quantitative change of $P(n)$ near the end of simulations between the transports from $W_{6}$ to $W_{5}$ and from $W_{10}$ to $W_{5}$, both for $\varepsilon=10^{-3}$, correspond to small statistical fluctuations.

Figure 9 illustrates the probability $P(n)$ for $\varepsilon=10^{-4}$, when the transport is from the origin window $W_{32}$ to the destination window $W_{31}$ and for $\varepsilon=10^{-3}$, when the transport is from $W_{9}$ to $W_{10}$. The upper inset of Fig. 9 displays, in log-linear scales, the exponential decay of $P(n)$ observed for small values of $n$. The lower inset of Fig. 9 displays, in log-linear scales, the behavior of $P(n)$ as function of $n^{1 / 2}$ in regime of large values of $n$. As we observe, in this regime, the plots of $P(n)$ present decays of stretched exponential type given by $P(n) \propto \exp (\delta \sqrt{n}), \delta<0$. For $\varepsilon=10^{-4}$ we have that, after $n^{1 / 2}=1.5 \times 10^{3}$, fewer than 80 trajectories of the initial $M=10^{6}$ remain in the simulation. The initial exponential decays observed in both curves are related to the contribution of the chaotic sea in the transport process while the stretched exponential decays are attributed to the temporary stick of trajectories near structures of regular motion [29].

Figure 10 displays the survival probability $P(n)$ as function of $n$ in log-linear scales. For $\varepsilon=10^{-3}$ the plot includes the transport of trajectories from the window of origin $W_{5}$ to the destination window $W_{6}$ and from $W_{5}$ to $W_{10}$. For $\varepsilon=10^{-4}$ we see the result of transport from $W_{31}$ to $W_{32}$. Looking closely at 


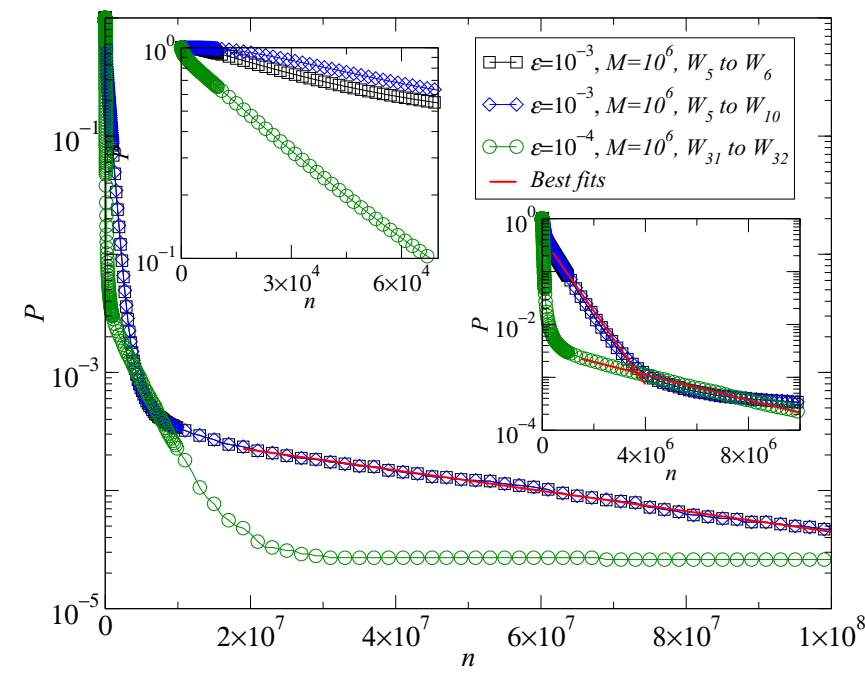

FIG. 10. Log-linear plot of survival probability for different values of parameter and different windows.

the curves of survival probability, we observe that $P(n)$ for the transport from $W_{5}$ to $W_{6}$ is, for each value of $n$, smaller than the probability for the transport from $W_{5}$ to $W_{10}$, as expected. For $\varepsilon=10^{-4}$, we observe that $P(n)$ is essentially constant after $n \approx 2 \times 10^{7}$, corresponding to fewer than 30 trajectories of the initial $M=10^{6}$. In all cases, after a brief transient, we observe for small-enough $n$ that $P(n)$ decays exponentially. This initial decay is presented for $\varepsilon=10^{-4}$ in the left inset of Fig. 10, while for $\varepsilon=10^{-3}$, and regarding both transports from $W_{5}$ to $W_{6}$ and from $W_{5}$ to $W_{10}$, the initial exponential decay is presented in the right inset of Fig. 10. For largeenough values of $n$ the probability curves bend towards the other regime of exponential decays characterized by smaller rates of decreasing than the former ones. The right inset of Fig. 10 illustrates this slower exponential decay observed for $\varepsilon=10^{-4}$, while for both transports associated to $\varepsilon=10^{-3}$, i.e., from $W_{5}$ to $W_{6}$ and from $W_{5}$ to $W_{10}$, this slower exponential

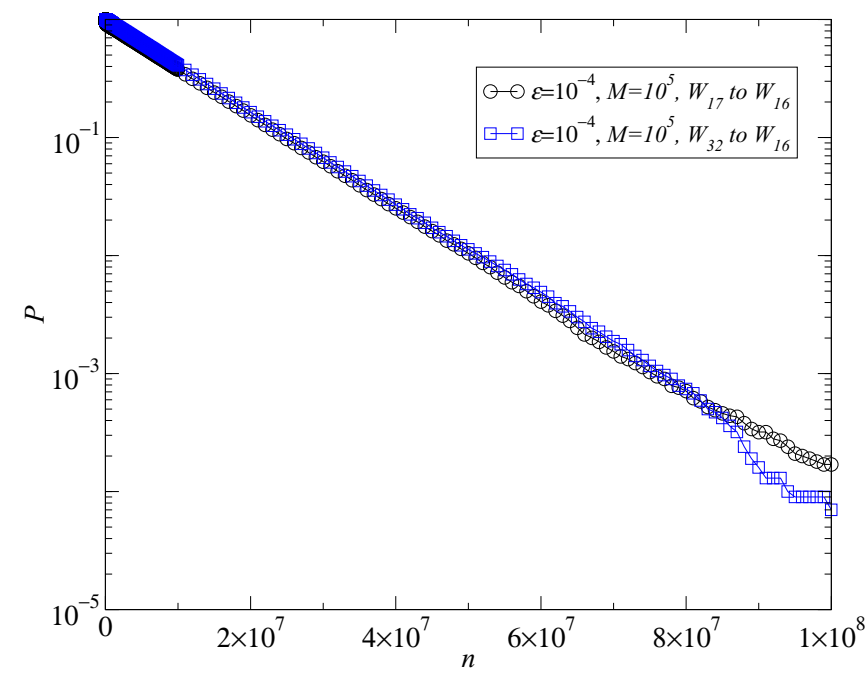

FIG. 11. Survival probability as function $n$ for the transport between different windows. decay is presented in the external plot of Fig. 10. Exponential decays observed in survival probability are associated to the contribution of the chaotic region of phase space $[6,25,26]$. So, in the cases presented here, the two different exponential decays observed in each curve are attributed to two groups of trajectories in the chaotic sea: (i) the initial, faster, exponential decays are related to trajectories that evolve more directly from $W_{o}$ to $W_{d}$ spending a short amount of time, while (ii) the later, slower, exponential decays are related to trajectories that evolve in a large portion of chaotic sea and spend more time before they reach the destination window.

Figure 11 illustrates the behavior of the survival probability for $\varepsilon=10^{-4}$ when the transport occurs from $W_{17}$ to $W_{16}$ and from $W_{32}$ to $W_{16}$. As we observe, in these cases the contribution of the chaotic sea is much more important than the contribution of the stick domains, because in each case the survival probability presents a single and exponential decay.

\section{CONCLUSIONS}

We presented the transport properties of chaotic trajectories in the phase space of the Fermi-Ulam model in two parts. In the first part we obtained the normalized histogram of transport and the survival probability for the transport of trajectories originating from a window located at a distance from the structures of regular motion. The destination window was defined as portions of chaotic sea above a velocity value established in terms of the positions of the lowest-energy-spanning curves. After scaling considerations, we demonstrated that the histogram for different values of $\varepsilon$ presents universal behavior. We showed also the survival probability presents an initial decay regime of exponential type followed by power-law decay for large $n$, in agreement with the mixed nature of phase space.

In the second part of this work we studied the transport process when the windows of origin are regions of phase space that enclose thin layers of the chaotic sea that surround islands of regular motion. The destination windows were defined in the same way. Regarding different combinations of windows of origin and destination, we demonstrated four kinds of behavior for the survival probability curves: (i) transition from an initial exponential decay to a power law decay, (ii) transition from an initial exponential decay to a regime of stretched exponential decay, (iii) transition from a fast exponential decay to a slower exponential decay, and (iv) a single and exponential decay. Cases (i) and (ii) are consistent with the mixed structure of phase space of the model under study. Because exponential decays in the survival probability indicate the contribution of chaotic motion in the transport process, the behavior described in case (iii) is attributed to two transport mechanisms. One of them is related to a set of trajectories that are quickly transported between the windows of origin and destination, generating the fast initial exponential decay. The other is attributed to a set of trajectories that evolve in a large portion of phase space and, consequently, spend more time before they reach the destination window, generating a slower exponential decay for large $n$. Despite the mixed structure of phase space, the behavior of survival probability described in cases (iii) and (iv) suggests the transport process is especially influenced by the chaotic portion of phase space, 
since power-law or stretched exponential decays are not observed.

Specifically for the last two cases above we can say that in a phase space of mixed type, where chaotic and regular trajectories coexist, depending on the particular choice of windows of origin (and destination), the transport process is weakly affected by the sticky domains associated to the structures of regular motion and strongly dominated by the chaotic portion of phase space.

Finally, the observation of two different regimes of exponential decay in the survival probability for a parameter value in the transport between two regions is surprising. According to our knowledge, this result is original and it opens a new perspective about the asymptotic behavior in transport processes in systems that present phase space with mixed structure. We intend to pursuit similar results and their consequences in future works for both one and twodimensional billiards.

\section{ACKNOWLEDGMENTS}

N.B.F. thanks FAPEMIG, D.S.T. thanks to CNPq, and D.G.L. thanks to CNPq (474674/2008-8) for the financial support. E.D.L. acknowledges Fapesp (2012/23688-5) and CNPq (303707/2015-1) for financial support.
[1] D. R. da Costa, M. R. Silva, and E. D. Leonel, Commun. Nonlin. Sci. Numer. Simulat. 19, 842 (2014).

[2] E. D. Leonel, D. R. da Costa, and C. P. Dettmann, Phys. Lett. A 376, 421 (2012).

[3] C. P. Dettmann, G. V. Morozov, M. Sieber, and H. Waalkens, Phys. Rev. A 80, 063813 (2009).

[4] E. G. Altmann, Phys. Rev. A 79, 013830 (2009).

[5] P. Gaspard, Chaos, Scattering and Statistical Mechanics (Cambridge University Press, Cambridge, 1998).

[6] E. Ott, Chaos in Dynamical Systems (Cambridge University Press, Cambridge, 2002).

[7] D. G. Ladeira and E. D. Leonel, Chaos 22, 043148 (2012).

[8] D. R. da Costa, I. L. Caldas, and E. D. Leonel, App. Math. Comput. 236, 215 (2014).

[9] A. L. P. Livorati, T. Kroetz, C. P. Dettmann, I. L. Caldas, and E. D. Leonel, Phys. Rev. E 86, 036203 (2012).

[10] T. E. Evans, R. A. Moyer, and P. Monat, Phys. Plasmas 9, 4957 (2002).

[11] J. S. E. Portela, I. L. Caldas, R. L. Viana, and M. A. F. Sanjuan, Int. J. Bifurcation Chaos Appl. Sci. Eng. 17, 4067 (2007).

[12] A. Wingen, M. Jakubowski, K. H. Spatschek, S. S. Abdullaev, K. H. Finken, M. Lehnen, and TEXTOR team, Phys. Plasmas 14, 042502 (2007).

[13] R. L. Viana, E. C. D. Silva, T. Kroetz, I. L. Caldas, M. Roberto, and M. A. F. Sanjuán, Philos. Trans. R. Soc. Lond. A 369, 371 (2011).

[14] W. Breymann, Z. Kovács, and T. Tél, Phys. Rev. E 50, 1994 (1994).

[15] L. G. G. V. D. da Silva and M. A. M. de Aguiar, Eur. Phys. J. B 16, 719 (2000).

[16] L. A. Bunimovich, Commun. Math. Phys. 65, 295 (1979).

[17] L. A. Bunimovich and Y. Sinai, Commun. Math. Phys. 78, 479 (1980).

[18] F. Vivaldi, G. Casati, and I. Guarneri, Phys. Rev. Lett. 51, 727 (1983).

[19] K.-C. Lee, Phys. Rev. Lett. 60, 1991 (1988).

[20] P. Gaspard and J. R. Dorfman, Phys. Rev. E 52, 3525 (1995).
[21] D. N. Armstead, B. R. Hunt, and E. Ott, Physica D 193, 96 (2004).

[22] L. A. Bunimovich, Chaos 11, 802 (2001).

[23] L. A. Bunimovich, Chaos 13, 903 (2003).

[24] L. A. Bunimovich, Discrete Contin. Dyn. Syst. 22, 63 (2008).

[25] T. Tél and M. Gruiz, Chaotic Dynamics (Cambridge University Press, Cambridge, 2006).

[26] E. G. Altmann, E. C. da Silva, and I. L. Caldas, Chaos 14, 975 (2004).

[27] C. F. F. Karney, Physica D 8, 360 (1983).

[28] B. V. Chirikov and D. L. Shepelyansky, Physica D 13, 395 (1984).

[29] C. P. Dettmann and E. D. Leonel, Physica D 241, 403 (2012).

[30] G. M. Zaslavsky, Phys. Rep. 371, 461 (2002).

[31] R. W. Easton, Trans. Am. Math. Soc. 294, 719 (1986).

[32] V. Rom-Kedar and S. Wiggins, Arch. Ration. Mech. Anal. 109, 239 (1990).

[33] J. D. Meiss, Chaos 25, 097602 (2015).

[34] H. E. Lomelí and J. D. Meiss, Chaos 10, 109 (2000).

[35] B. V. Chirikov and D. L. Shepelyansky, Phys. Rev. Lett. 82, 528 (1999).

[36] N. N. Thyagu and N. Gupte, Phys. Rev. E 79, 066203 (2009).

[37] C. P. Dettmann and O. Georgiou, Physica D 238, 2395 (2009).

[38] C. P. Dettmann and O. Georgiou, Phys. Rev. E 83, 036212 (2011).

[39] R. S. MacKay, J. D. Meiss, and I. C. Percival, Physica D 13, 55 (1984).

[40] H. E. Lomelí and J. D. Meiss, Nonlinearity 22, 1761 (2009).

[41] J. D. Mireles-James, J. Nonlin. Sci. 23, 585 (2013).

[42] A. J. Lichtenberg and M. A. Lieberman, Regular and Chaotic Dynamics, 2nd ed., Appl. Math. Sci., Vol. 38 (Springer-Verlag, New York, 1992).

[43] D. R. da Costa, C. P. Dettmann, and E. D. Leonel, Phys. Rev. E 83, 066211 (2011).

[44] A. J. Lichtenberg, M. A. Lieberman, and R. H. Cohen, Physica D 1, 291 (1980).

[45] E. D. Leonel, J. K. L. da Silva, and S. O. Kamphorst, Physica A 331, 435 (2004). 